Open Access

\title{
Well-differentiated angiosarcoma of spleen: a teaching case mimicking hemagioma and cytogenetic analysis with array comparative genomic hybridization
}

Lichen $\mathrm{Xu}^{1+}$, Yimin Zhang ${ }^{1+}$, Hong Zhao ${ }^{1}$, Qingxiao Chen ${ }^{2}$, Weihang $\mathrm{Ma}^{1,3^{*}}$ and Lanjuan $\mathrm{Li}^{1}$

\begin{abstract}
Primary splenic angiosarcoma is extremely rare but aggressive malignant vascular neoplasm. Here, we report a case of vascular tumor in spleen that was initially misinterpreted as hemangioma. Two years after splenectomy, the patient admitted again with aggravated abdomen pain and severe anemia. The magnetic resonance imaging (MRI) scan showed widely metastases. The ensuing biopsy for lesion both in liver and in bone marrow showed the similar pathological findings as that in spleen, which supported the final diagnosis of well-differentiated splenic angiosarcoma with extensive metastases. The patient was dead in 3 months after discharge without chemotherapy. The copy number changes for spleen lesion detected by array comparative genome hybridization showed copy number gain at 11q23.2, 11q24.3, 12q24.33, 13q34, copy number loss at 1q24.2-q31.3, 1q41-q42.2, 1 q42.3-q43, 2q36.3-q37.3, 2q37.7, 3q13.33-q26.2, 3q28 - q29, 9p11.2, 13q11, 15q11, homozygous copy loss at 8p11.22, 22q11.23. Less than 200 cases of splenic angiosarcoma have been published in literature of English. To the best of our knowledge, this is the first time analyzed cytogenetic alteration in a well-differentiated primary splenic angiosarcoma.
\end{abstract}

Keywords: Angiosarcoma, Well-differentiation, Splenectomy, Array comparative genomic hybridization, Copy number change

\section{Background}

Primary splenic angiosarcoma (PSA) is a rare malignant neoplasm of endothelial cell, originating from vascular or lymphatic. PSA was first reported by Langhans in 1879. To date, no more than 200 cases of splenic angiosarcoma have been reported in literature [1]. The morphology features for this tumor are often uncharacteristic, ranging from well-formed anastomosing vessels to high-grade epithelioid or spindled cells without clear vasoformation [2]. Moreover, multiple appearances can be seen in the same case, making great challenge for diagnosis. However, no comprehensive research of

\footnotetext{
* Correspondence: weihangma2014@126.com

${ }^{\dagger}$ Equal contributors

${ }^{1}$ State Key Laboratory for Diagnosis and Treatment of Infectious Diseases, Collaborative Innovation Center for Diagnosis and Treatment of Infectious Diseases, The First Affiliated Hospital, College of Medicine, Zhejiang University, 79 Qingchun Road, Hangzhou, Zhejiang 310003, China ${ }^{3}$ School of Medicine, Zhejiang University, Hangzhou, Zhejiang, China Full list of author information is available at the end of the article
}

cytogenetic changes to this subgroup of angiosarcoma has been published.

In this article, we describe a 38-year-old female patient of malignant vascular tumor that was initially interpreted as benign hemangioma in spleen with subtle cytological atypia and died 2 years later for multiple organ metastases. At the same time, we provided further array comparative genome hybridization (aCGH) analysis to make a preliminary exploration on the earlier identification of subgroup of angiosarcoma. To date, on cytogenetic analysis specific for primary splenic angiosarcoma has been reported.

\section{Case presentation}

A 38-year-old female patient was admitted to the hospital after suffering upper abdomen pain and fatigue for about 1 week in 2011. She has no history of smoking and alcohol or drug abuse. The patient denied immunodeficiency, history of malignancy and toxin exposure, and family 
history of genetic disorders. The abdomen computed tomography (CT) (Fig. 1) has been performed for further examination. The result showed splenomegaly with multifocal low-density lesions. Other organs including liver, pancreas, renal, and adrenal were normal. Considering possibility of splenic neoplasm, and for further clarification, splenectomy was carried out. Histopathology (Fig. 2) evaluation showed well-formed anastomosing vessel channels and proliferated endothelial cell with mild atypia but inconspicuously malignant. Immunohistochemistry (Fig. 2) revealed positive staining of CD31and CD34 but negative staining of factor VIII, CD68, p53. The pathology findings supported the diagnosis of hemangioma. Although no necrosis or hemorrhage was found in macroscopic and histological examination, in consideration of splenomegaly and anastomosing capillary-sized vessels, the well-differentiated angiosarcoma cannot be excluded. After splenectomy, the patient was quickly relieved from the pain and discharged without any postoperative complications. Then, the patient got lost to follow-up.

Two years after splenectomy in December 2013, the patient presented with worsening upper abdomen pain, fatigue, and frequent dizziness for 3 months. On physical examination, she showed severe anemic appearance. There were no clinically palpable node and no papilledema. There was no evidence of bruising and easy bleeding. The initial lab tests were as follows: low hemoglobin (45 g/L, normal range 113-151), an increase of D-dimer, 78,700 ug/L (normal range 0-700ug/L), and a mild increase of aspartate aminotransferase (AST), $48 \mathrm{U} / \mathrm{L}$ (normal range 8-40U/L). Further examination of upper abdominal magnetic resonance imaging (MRI) was implemented. The result (Fig. 3) showed multiple nodes in the liver and accessory spleen with rich blood supply and signals of intratumoral hemorrhage. With these findings in MRI, fine-needle biopsy was obtained of the hepatic lesions. Due to further clarification of anemia, bone marrow trephine biopsy as well as smear for cytological examination were performed. The pathology evaluation (Fig. 2) on hepatic demonstrated a small amount of proliferated endothelial cells. The result of pathology findings of bone marrow biopsy (Fig. 2) showed anastomosing vascular channels, proliferated endothelial cells with few mitotic figures and mild nuclear atypia. The similarity of pathological findings in the liver, spleen, and bone marrow and clinical image presentation supported that the final diagnosis was well-differentiated angiosarcoma of the spleen with bone and liver metastasis. The patient was initially treated with analgesia and blood transfusion. Because few data can be acquired to guide chemotherapy and poor therapeutic effect, she gave up further chemotherapy after discussing with her family members. She was
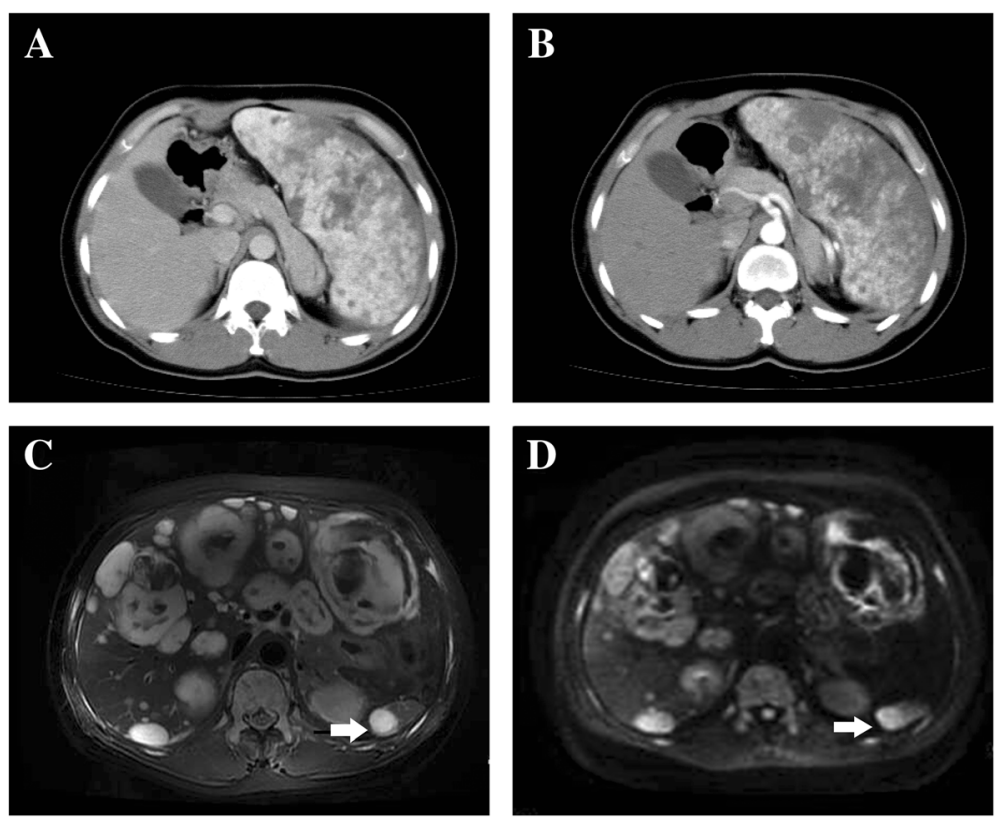

Fig. 1 Abdomen CT scan in 2011 (a, b) demonstrated splenomegaly and multiple irregular low-density lesions. Liver, renal, adrenal, and pancreas are normal. The MRI in 2013 presented multiple cystic lesions in different sizes diffused in liver. The lesions displayed as inhomogeneous mixed signal, with high signal intensity on T2-weighted image (T2WI, c) and diffusion-weighted image (DWI, d). Some lesions that indicated eccentric or central low signal intensity were suspected intertumoral hemorrhage. Nodule lesion with abnormal sign as that in the liver was also found in remnant accessory spleen (arrow) 

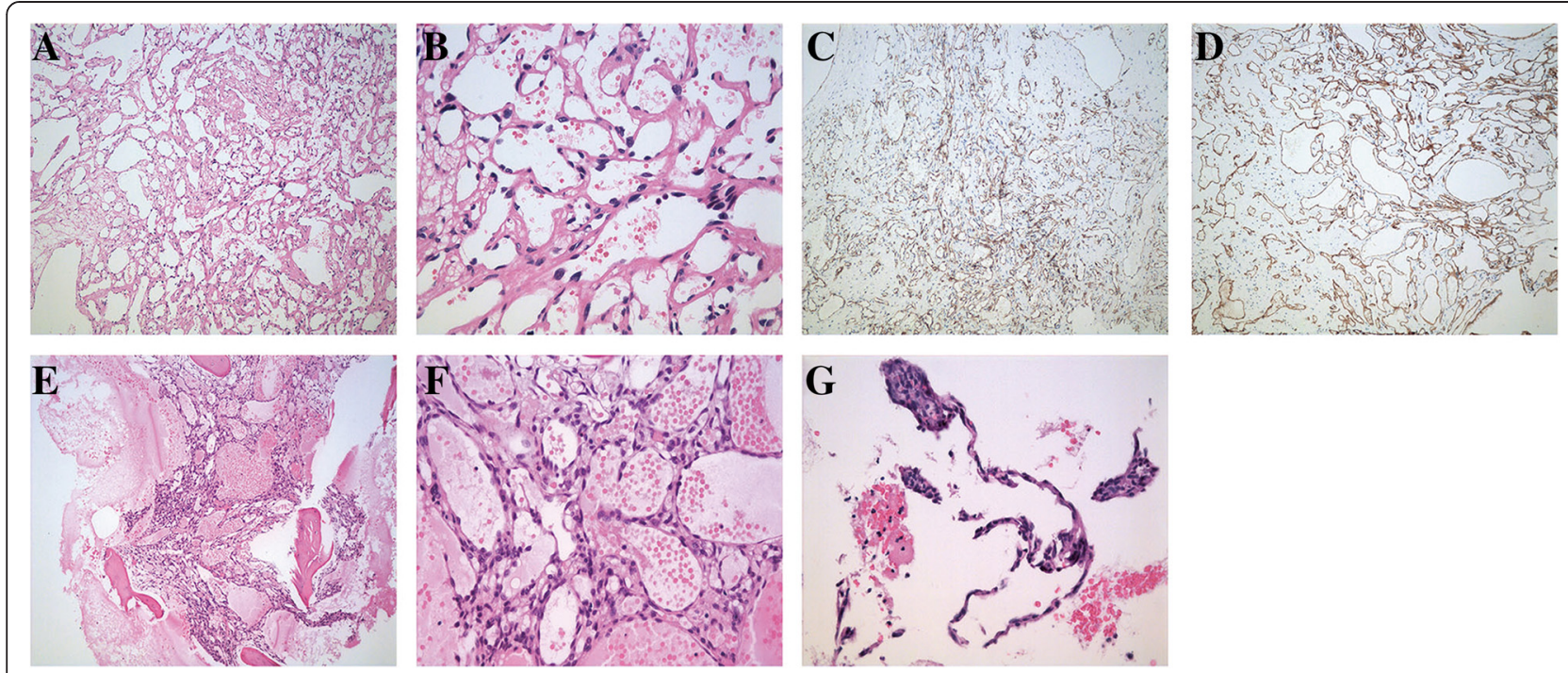

Fig. 2 Tumor in spleen shows well-formed anastomosing vessel channels (a, HE 100x), proliferated endothelial cells with mild atypia (b, HE 400x), immunohistochemistry revealed positive staining of CD31 (c, 100x) and CD34 (d, 100x). Tumor cells from bone biopsy showed anastomosing vessel channels with proliferated endothelial cells without obvious malignant as that in spleen (e, HE 100x; f, HE 400x). Tumor cells from liver biopsy showed a handful of endothelial cells without obvious malignant (g, HE 100x)

discharged in a few days after slight relief from pain and fatigue. Follow-up showed she passed away 3 months after discharge.

DNA was extracted from ten $20-\mu \mathrm{m}$ thick ribbons of paraffin-embedded tumor of spleen from FFPE tissue blocks using DNeasy Blood \&Tissue Kit (Cat\#69506, QIAGEN, GmBH, Germany), following the manufacturer's instructions. A total of $80 \mathrm{ng}$ sample of DNA was sent for processing via OconScanTM FFPE Express 2.0 service as manufacturer's instructions. The OncoScan assay contains more than 300,000 copy number and single nucleotide polymorphism (SNP) oligonucleotide probes with a median probe spacing of $4200 \mathrm{~kb}$, with 541 somatic mutations for known cancer genes. The arrays were scanned by GeneChip Scanner 3000 (Cat\#00-00212, Affymetrix, Santa Clara, CA, USA) and Command Console Software 3.1 (Affymetrix, Santa Clara, CA, USA) with default settings. Raw data that passed quality control were further analyzed by Affymetrix ${ }^{\circ}$ OconScan Analysis Suite (Affymetrix, Santa Clara, CA, USA).

Statistical analysis of the CGH data was performed by FASST2 Segmentation method. In order to adjust the sensitivity of the segmentation algorithm, we determined the significant threshold at $1.0 \mathrm{E}-8$, specified $1000 \mathrm{~kb}$ being max contiguous probe spacing. The minimum number of probes per segment required to

Fig. 3 Summary of chromosomes copy number changes of splenic angiosarcoma detected by aCGH. The blue color on the right side represents


eliminate small CNVs was five; gains and losses were defined at $\pm 3 \times \mathrm{SD}$ of all probes, and the threshold was adjusted at \pm 0.5 for both.

Copy number change of this angiosarcoma of spleen sample showed diverse DNA copy number alternations including copy number loss, copy number gain, and homozygous copy loss. The copy number changes were summarized in Fig. 3 and Table 1. The result (Fig. 4 and Table 1) revealed that copy number gain at $11 \mathrm{q} 23.2,11 \mathrm{q} 24.3,12 \mathrm{q} 24.33,13 \mathrm{q} 34$, copy number loss at 1q24.2-q31.3, 1q41-q42.2, 1 q42.3-q43, 2q36.3q37.3, 2q37.7, 3q13.33-q26.2, 3q28-q29, 9p11.2, 13q11, $15 \mathrm{q} 11$, homozygous copy loss at 8p11.22, 22q11.23.

We found that a total of 1067 genes were affected by copy number changes according to the result of aCGH. In order to discuss the probably functions of altered genes, 397 out of 1067 genes with known biological functions, we used the Database for Annotation, Visualization and Integrated Discovery (DAVID) v6.7 (free online bioinformatics resources at http:// david.abcc.ncifcrf.gov/) to clustered Gene Ontology (GO) group. The enriched GO groups (Fig. 4) were ranked according to statistical significance measured by EASE score (EASE score $=-\mathrm{LgP})$, a modified Fish's exact $p$ value. The result illustrates that five relevant pathways have been affected by copy number changes as EASE score upper than 0.1 . It is noteworthy that renin-angiotensin system and ECM-receptor interaction pathways exhibit statistically EASE score $(p<0.05)$.

\section{Discussion}

Angiosarcoma, an extremely rare vascular malignant tumor, approximately composes $2 \%$ of all soft tissue sarcoma. The incidence rate has risen over the past 30 years with unclear causes [3, 4]. PSA is the rare type of angiosarcoma. A tumor distribution analysis of 543 patients with angiosarcoma indicated PSA account for $2.5 \%$ [3]. Clinical manifestation among PSA patient is variable and unspecific. Abdominal pain, weakness or fatigue, fever, chest pain, and/or weight loss, anemia, and thrombocytopenia were relatively predominant clinical presentation. Another, the sign related to metastasis like short of breath and gastrointestinal bleeding also can be the starting clinical manifestation when a patient was admitted [5-8]. Splenomegaly was commonly depicted in physical examination, and it can even be the only finding [6]. Some patients presented with celiac hemorrhage due to spontaneous splenic rupture, which occurred 13-32\% in the literature [6,9]. Chemotherapeutic efficacy is still unclear but splenectomy as early as possible in people without metastasis may prolong period of survival [10].

Early metastasis was often taken place in high incidence with mostly involved in liver, bone marrow, lung, and lymph nodes. Aggressive progress and early metastasis always lead to dismal prognosis in patients with PAS. Unfortunately, histological findings cannot fully predict its malignant clinical behavior that even with brisk atypical histological changes may present metastatic lesions [11, 12]. Molecular tests have long been

Table 1 Chromosomes copy number changes of splenic angiosarcoma

\begin{tabular}{|c|c|c|c|c|}
\hline Chromosome & Location & Evens & Region length (bp) & Gene number \\
\hline \multicolumn{5}{|c|}{ (a) Copy number (CN) loss or homozygous copy loss } \\
\hline chr1 & $q 24.2-q 31.3$ & CN loss & 30010137 & 238 \\
\hline chr1 & $q 41-q 42.2$ & CN loss & 15946527 & 156 \\
\hline chr1 & $q 42.3-q 43$ & CN loss & 19069070 & 21 \\
\hline chr2 & q36.3-q37.3 & CN loss & 8571661 & 101 \\
\hline chr2 & q37.7 & CN loss & 4012418 & 71 \\
\hline chr3 & q13.33-q26.2 & CN loss & 50643792 & 428 \\
\hline chr3 & q28-q29 & CN loss & 2176220 & 9 \\
\hline chr8 & p11.22 & Homozygous copy loss & 197795 & 3 \\
\hline chr9 & $\mathrm{p} 11.2$ & $\mathrm{CN}$ loss & 794282 & 1 \\
\hline chr13 & $q 11$ & CN loss & 289503 & 2 \\
\hline chr15 & $q 11.1$ & CN loss & 51427 & 0 \\
\hline chr22 & $q 11.23$ & Homozygous copy loss & 43891 & 4 \\
\hline \multicolumn{5}{|c|}{ (b) Copy number gain } \\
\hline chr11 & q23.2 & CN gain & 174597 & 1 \\
\hline chr11 & q24.3 & CN gain & 427283 & 7 \\
\hline chr12 & $\mathrm{q} 24.33$ & CN gain & 681673 & 20 \\
\hline chr13 & q34 & CN gain & 520705 & 5 \\
\hline
\end{tabular}




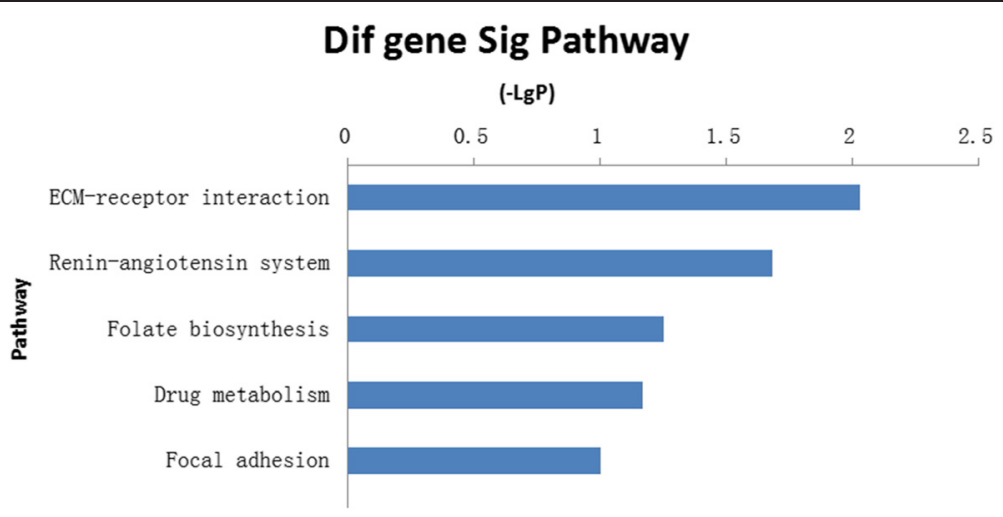

Fig. 4 Gene ontology groups enriched by genes with copy number losses and gains. EASE score higher than 0.1 has been listed in the figure. The gene functional analysis revealed enrichments for otology terms related to diverse pathways, involved renin-angiotensin system and ECM-receptor interaction pathways signaling pathways

needed for diagnosis and classification. However, cytogenetic information on chromosomal abnormal on angiosarcomas are limited. In most cases, cytogenetic analysis indicated complex karyotypes, and the specific aberration characteristics for tumor development and behavior have far not been identified [13]. Gains in 8q and 20p and losses in 22q are the most frequent chromosomes imbalances among angiosarcomas [14]. But it should be kept in mind that the angiosarcomas in different anatomical sites or in different clinical settings might be based on distinguishing gene-expression profiling. A novel (1:14) (p21:q24) translocation has been reported in a primary bone angiosarcoma [15]. Cluster analysis of aCGH has identified two subgroup of primary bone angiosarcoma: a group with a complex genetic profile and a group with only few genetic aberrations [16]. Recent studies have shown that high-level amplification of MYC oncogene on chromosome 8q24.21 is specific for secondary angiosarcoma after radiation or lymphedema [17]. Co-amplification of FLT4 (encoding VEGFR3) on 5 q35 was detected in $25 \%$ of radiation-induced angiosarcoma [18]. Both FLT4 and MYC gene abnormalities can potentially be used as molecular diagnostic tool to distinguish from atypical vascular lesions (AVL) and secondary angiosarcoma $[18,19]$. Loss of heterozygosity $(\mathrm{LOH})$ of WT-1, RB1, and P53 locus at chromosome region 11p13, $13 q 14$, and 17p13, respectively, was reported in angiosarcoma, supporting the role of those tumor suppressor genes in pathogenesis of angiosarcoma [20].

In the present case, we applied Affymetrix OncoScanTM FFPE Express molecular inversion probe microarray platform to analyze the chromosome abnormal. The result in this case was the same as reported elsewhere that angiosarcoma often displayed complex chromosome aberration [13]. The previous researches that benign vascular neoplasm often shows simple chromosome abnormality may support the malignant nature of the present case [21]. Notably, this observation raises possibility that even well-differential angiosarcoma may presents complex chromosome abnormality.

Aberration of 11q24.3 as seen in this case has reported in several malignancy. But the molecular mechanism in PAS remains unknown. Copy number gain in 11q24.3 may result in a constitutive high expression of ETS1 and FLI1 genes. EST1 was found up-regulated a number of angiogenic factors, such as vascular endothelial growth factor (VEGF), basic fibroblast growth factor (bFGF), angiotensin II, endothelin-1, tumor necrosis factor- $\alpha$ (TNF- $\alpha$ ), or hydrogen peroxide. EST1 and FLI genes, ETS transcription factors, have been proved to play a significant role in malignancies [22, 23]. Conversely, inhibition of EST1 expression reduces the ability of endothelial cells to migrate, to proliferate, or to adopt an invasive behavior [23]. Strong expression of EST1 protein have been observed in angiosarcoma of skin, while it weakens in hemangioma [24]. FLI expression is a highly sensitive marker of a wide variety of common and unusual vascular tumors, but it cannot distinguish benign and malignant. A recent comprehensive study in diffuse large B cell lymphoma showed copy number gain in $11 \mathrm{q} 24.3$ and constructively up-regulates EST1 and FLI genes, which contributes to the pathogenesis of disease [25]. However, the roles of 11q24.3 amplification in PAS require further research both in molecular mechanism and in large sample investigation.

\section{Conclusions}

In summary, primary splenic angiosarcoma remains to be a rare neoplasm with a high propensity for metastasis. It is still different to diagnose according to histopathology findings and immunohistochemistry profiles. But combined with radiological manifestation and microscopic examination, it may benefit to make final diagnosis. 
Cytogenetic analysis also gives some help to trace the malignant nature.

To the best of our knowledge, this is the first time that cytogenetic alteration was analyzed in a well-differentiated primary splenic angiosarcoma. However, the molecular features to characterize this subgroup of angiosarcoma need larger scale and more comprehensive study.

\section{Consent}

The patient and the families were informed that data from the case would be submitted for publication and provided their consent accordingly.

\section{Abbreviations}

MRI: magnetic resonance imaging; PSA: primary splenic angiosarcoma; aCGH: array comparative genome hybridization; CT: computed tomography; AST: aspartate aminotransferase; DAVID: database for annotation, visualization and integrated discovery; LOH: loss of heterozygosity; VEGF: vascular endothelial growth factor; bFGF: basic fibroblast growth factor; TNF-a: tumor necrosis factor-a; AVL: atypical vascular lesions.

\section{Competing interests}

The authors declare that they have no competing interests.

\section{Authors' contributions}

$L X$ wrote this case report. LX and $Y Z$ participated in the design of the study and analyzed the data. WM conceived the study and participated in its design and coordination. WM and LL helped draft the manuscript. QC provided the photos and artworks. All authors read and approved the final manuscript.

\section{Acknowledgements}

This work was supported by Medical science and technology project of Zhejiang Province (2013KYB084).

\section{Author details}

${ }^{1}$ State Key Laboratory for Diagnosis and Treatment of Infectious Diseases, Collaborative Innovation Center for Diagnosis and Treatment of Infectious Diseases, The First Affiliated Hospital, College of Medicine, Zhejiang University, 79 Qingchun Road, Hangzhou, Zhejiang 310003, China. ${ }^{2}$ Bone Marrow Transplantation Center, Department of Hematology, School of Medicine, Zhejiang University, Hangzhou, Zhejiang, China. ${ }^{3}$ School of Medicine, Zhejiang University, Hangzhou, Zhejiang, China.

Received: 29 July 2015 Accepted: 5 October 2015

Published online: 13 October 2015

\section{References}

1. Hamid KS, Rodriguez JA, Lairmore TC. Primary splenic angiosarcoma. JSLS. 2010;14(3):431-5.

2. Antonescu C. Malignant vascular tumors_an update. Mod Pathol. 2014;27 Suppl 1:S30-8.

3. Young RJ, Brown NJ, Reed MW, Hughes D, Woll PJ. Angiosarcoma. Lancet Oncol. 2010;11(10):983-91.

4. Rouhani P, Fletcher CD, Devesa SS, Toro JR. Cutaneous soft tissue sarcoma incidence patterns in the U.S.: an analysis of 12,114 cases. Cancer. 2008;113(3):616-27.

5. Hsu JT, Lin CY, Wu TJ, Chen HM, Hwang TL, Jan YY. Splenic angiosarcoma metastasis to small bowel presented with gastrointestinal bleeding. World J Gastroenterol. 2005;11(41):6560-2.

6. Neuhauser TS, Derringer GA, Thompson LDR, Fanburg-Smith JC, Miettinen M, Saaristo A, et al. Splenic angiosarcoma: a clinicopathologic and immunophenotypic study of 28 cases. Modern Pathol. 2000;13(9):978-87.

7. Qi R, Yu JQ, Xu H, Zhou XP, Li XM. Primary angiosarcoma of the spleen as depicted on computed tomography. Clin Imag. 2012;36(5):619-22.

8. Hsu JT, Chen HM, Lin CY, Yeh CN, Hwang TL, Jan YY, et al. Primary angiosarcoma of the spleen. J Surg Oncol. 2005;92(4):312-6.
9. Falk S, Krishnan J, Meis JM. Primary angiosarcoma of the spleen-a clinicopathological study of 40 cases. Am J Surg Pathol. 1993;17(10):959-70.

10. Liu ZJ, Du XF, Li HT, Wang ZY, Shen Z, Yao Y, et al. Primary splenic angiosarcoma. VASA. 2012;41(1):57-62.

11. Fernandez S, Cook GW, Arber DA. Metastasizing splenic littoral cell hemangioendothelioma. Am J Surg Pathol. 2006;30(8):1036-40.

12. Ben-Izhak O, Bejar J, Ben-Eliezer S, Vlodavsky E. Splenic littoral cell haemangioendothelioma: a new low-grade variant of malignant littoral cell tumour. Histopathology. 2001;39(5):469-75.

13. Baumhoer D, Gunawan B, Becker H, Fuzesi L. Comparative genomic hybridization in four angiosarcomas of the female breast. Gynecol Oncol. 2005;97(2):348-52. doi:10.1016/j.ygyno.2004.12.046.

14. Mertens F, Panagopoulos I, Mandahl N. Genomic characteristics of soft tissue sarcomas. Virchows Arch. 2010;456(2):129-39.

15. Dunlap JB, Magenis RE, Davis C, Himoe E, Mansoor A. Cytogenetic analysis of a primary bone angiosarcoma. Cancer Genet Cytogenet. 2009;194(1):1-3.

16. Verbeke SL, de Jong D, Bertoni F, Sciot R, Antonescu CR, Szuhai K, et al. Array $\mathrm{CGH}$ analysis identifies two distinct subgroups of primary angiosarcoma of bone. Genes Chromosomes Cancer. 2015;54(2):72-81.

17. Manner J, Radlwimmer B, Hohenberger P, Mossinger K, Kuffer S, Sauer C, et al. MYC high level gene amplification is a distinctive feature of angiosarcomas after irradiation or chronic lymphedema. Am J Pathol. 2010;176(1):34-9.

18. Guo TH, Zhang L, Chang NE, Singer S, Maki RG, Antonescu CR. Consistent MYC and FLT4 gene amplification in radiation-induced angiosarcoma but not in ther radiation-associated atypical vascular lesions. Gene Chromosome Canc. 2011;50(1):25-33.

19. Fernandez AP, Sun Y, Tubbs RR, Goldblum JR, Billings SD. FISH for MYC amplification and anti-MYC immunohistochemistry: useful diagnostic tools in the assessment of secondary angiosarcoma and atypical vascular proliferations. J Cutan Pathol. 2012;39(2):234-42.

20. Domfeh $A B$, Fichera M, Hunt JL. Allelic loss of 3 different tumor suppressor gene loci in benign and malignant endothelial tumors of the head and neck. Arch Pathol Lab Med. 2006;130(8):1184-7.

21. Wen WW, MacKenzie KL. Modeling human endothelial cell transformation in vascular neoplasias. Dis Model Mech. 2013;6(5):1066-79.

22. Zhang XK, Gallant S, Molano I, Moussa OM, Ruiz P, Spyropoulos DD, et al. Decreased expression of the Ets family transcription factor Fli-1 markedly prolongs survival and significantly reduces renal disease in MRL/lpr mice. J Immunol. 2004;173(10):6481-9.

23. Dittmer J. The biology of the Ets1 proto-oncogene. Mol Cancer. 2003;2:29.

24. Naito $S$, Shimizu $K$, Nakashima M, Nakayama T, Ito $T$, Ito $M$, et al. Overexpression of Ets-1 transcription factor in angiosarcoma of the skin. Pathol Res Pract. 2000;196(2):103-9.

25. Bonetti $\mathrm{P}$, Testoni M, Scandurra M, Ponzoni M, Piva R, Mensah AA, et al. Deregulation of ETS1 and FLI1 contributes to the pathogenesis of diffuse large B-cell lymphoma. Blood. 2013;122(13):2233-41.

\section{Submit your next manuscript to BioMed Central and take full advantage of:}

- Convenient online submission

- Thorough peer review

- No space constraints or color figure charges

- Immediate publication on acceptance

- Inclusion in PubMed, CAS, Scopus and Google Scholar

- Research which is freely available for redistribution 